\title{
A Refined Multiscale Self-Entropy Approach for the Assessment of Cardiac Control Complexity: Application to Long QT Syndrome Type 1 Patients
}

\author{
Vlasta Bari ${ }^{1}$, Giulia Girardengo ${ }^{2}$, Andrea Marchi ${ }^{3,4}$, Beatrice De Maria ${ }^{5}$, \\ Paul A. Brink ${ }^{6}$, Lia Crotti ${ }^{2,7}$, Peter J. Schwartz ${ }^{2}$ and Alberto Porta ${ }^{1,8, *}$
}

1 Department of Biomedical Sciences for Health, University of Milan, Via F. Fellini 4, 20097 San Donato Milanese, Italy; E-Mail: vlasta.bari@grupposandonato.it

2 Center for Cardiac Arrhythmias of Genetic Origin, IRCCS Istituto Auxologico Italiano, Centro Diagnostico San Carlo, Via Pier Lombardo 22, 20135 Milan, Italy;

E-Mails: giuliagira82@yahoo.it (G.G.); liacrotti@yahoo.it (L.C.); peter.schwartz@unipv.it (P.J.S.)

3 Department of Electronics Information and Bioengineering, Politecnico di Milano, Piazza Leonardo da Vinci 32, 20133 Milan, Italy; E-Mail: marchi.andrea1@gmail.com

4 Department of Emergency and Intensive Care, San Gerardo Hospital, 20900 Monza, Italy

5 IRCCS Fondazione Salvatore Maugeri, 20138 Milan, Italy; E-Mail: beatrice.demaria@fsm.it

6 Department of Internal Medicine, University of Stellenbosch, Stellenbosch 7602, South Africa; E-Mail: pab@sun.ac.za

7 Institute of Human Genetics, Helmholtz Zentrum München, Ingolstädter Landstraße 1, 85764 Neuherberg, Germany

8 Department of Cardiothoracic, Vascular Anesthesia and Intensive Care, IRCCS Policlinico San Donato, Via Morandi 30, 20097 San Donato Milanese, Italy

* Author to whom correspondence should be addressed; E-Mail: alberto.porta@unimi.it; Tel.: +39-02-52774382; Fax: +39-02-50319979.

Academic Editor: Anne Humeau-Heurtier

Received: 14 September 2015 / Accepted: 13 November 2015 / Published: 19 November 2015

\begin{abstract}
The study proposes the contemporaneous assessment of conditional entropy (CE) and self-entropy (sE), being the two terms of the Shannon entropy (ShE) decomposition, as a function of the time scale via refined multiscale CE (RMSCE) and sE (RMSsE) with the aim at gaining insight into cardiac control in long QT syndrome type 1 (LQT1) patients featuring the KCNQ1-A341V mutation. CE was estimated via the corrected CE (CCE) and $\mathrm{sE}$ as the difference between the ShE and CCE. RMSCE and
\end{abstract}


RMSsE were computed over the beat-to-beat series of heart period (HP) and QT interval derived from 24-hour Holter electrocardiographic recordings during daytime (DAY) and nighttime (NIGHT). LQT1 patients were subdivided into asymptomatic and symptomatic mutation carriers (AMCs and SMCs) according to the severity of symptoms and contrasted with non-mutation carriers (NMCs). We found that RMSCE and RMSsE carry non-redundant information, separate experimental conditions (i.e., DAY and NIGHT) within a given group and distinguish groups (i.e., $\mathrm{NMC}, \mathrm{AMC}$ and $\mathrm{SMC}$ ) assigned the experimental condition. Findings stress the importance of the joint evaluation of RMSCE and RMSsE over HP and QT variabilities to typify the state of the autonomic function and contribute to clarify differences between AMCs and SMCs.

Keywords: information storage; heart rate variability; QT interval; refined multiscale entropy; LQT1; corrected conditional entropy; KCNQ1-A341V mutation; autonomic nervous system

\section{Introduction}

Information domain analysis of time series provides relevant information about the behavior of complex physiological systems [1-7]. Indeed, in this domain indexes of dynamical complexity are naturally quantified via self-entropy $(\mathrm{sE})$ and conditional entropy $(\mathrm{CE})$, measuring the portions of the amount of information about the current variable that can and cannot be derived from previous past values of the same variable respectively. The higher the $\mathrm{sE}$, the higher the predictability and regularity of the series is. The higher the CE, the higher the complexity of the series is. CE and sE can be seen as portions of the decomposition of the overall amount of information carried by a series given that their sum is the Shannon entropy (ShE) [7-10]. While the CE is more widely utilized as a measure of complexity of a series [1-3], sE is traditionally exploited to assess regularity and predictability of a process [8] or information stored in it [7,9].

CE depends on time scales and this dependence, usually assessed via multiscale conditional entropy (MSCE) approach [11] or via its refinement referred to as refined MSCE (RMSCE) [12], provides relevant information about cardiovascular regulation because it allows the focalization of cardiac control mechanisms acting over an assigned time scale [11-17]. While the importance of monitoring the $\mathrm{CE}$ as a function of the time scale via MSCE or RMSCE is indubitable, it is unclear whether sE is worth to be monitored as a function of the time scale. It can be hypothesized that the course of sE as a function of the time scale carries complementary information compared to MSCE or RMSCE in patho-physiological conditions.

The aim of this work is to compute $\mathrm{CE}$ and $\mathrm{sE}$ as a function of the time scale via RMSCE and refined multiscale sE (RMSsE) approaches and to check their ability and non-redundant value in describing cardiac control. RMSCE and RMSsE were evaluated over heart period (HP) and QT interval series derived from 24-hour Holter electrocardiographic recordings in a long QT syndrome type 1 (LQT1) founder population featuring the KCNQ1-A341V mutation. LQT1 mutation carriers (MCs) were subdivided into asymptomatic MCs (AMCs) and symptomatic MCs (SMCs) based on the 
severity of the symptoms and different arrhythmic risk, and contrasted to non MCs (NMCs) belonging to the same family line $[18,19]$.

\section{Methods}

\subsection{MSCE and RMSCE}

MSCE estimates complexity of a time series $x=\left\{x_{i}, i=1, \ldots, N\right\}$, where $i$ is a progressive counter and $N$ is the series length, via an entropy rate (e.g., CE) after low pass filtering focusing progressively slower time scales [11]. Such a method consists in low pass filtering $x$ to focus the range of time scales of interest while discarding the fastest ones, downsampling the filtered version according to the cut-off of the low pass filter utilized to eliminate fast components and assessing CE over the low pass filtered version of $x$. The filtering procedure, approximately preserving oscillations of $x$ with frequencies from 0 to $1 / \tau$, where $\tau$ is an integer value denoted as time scale, is iteratively applied to the filtered version, thus progressively eliminating lower and lower frequencies and focusing on dynamics with longer and longer time scales. The original procedure to compute the MSCE was refined to limit its dependence on the variance of $x$ and to more efficiently prevent aliasing during the downsampling procedure [12]. Such a refinement was achieved by updating the tolerance for the calculation of the CE to account for the eventual reduction of the variance of $x$ resulting from the filtering procedure and by substituting the suboptimal original low pass filter performing the mere mean at any step of the procedure with a Butterworth filter.

\subsection{Estimate of RMSCE and RMSSE}

In the MSCE approach the CE, usually computed as the sample entropy [2], is not the unique information-theoretic quantity that deserves to be monitored over $x$ as a function of the time scale. Indeed, another notable information-theoretic quantity that can be computed in univariate applications, denoted as $\mathrm{sE}$, received a lot of attention for its ability to quantify regularity [8] and information storage $[7,9]$.

Let us define a pattern as an ordered sequence of $L$ samples $\boldsymbol{x}_{i}^{L}=\left[x_{i}, x_{i-1}, \ldots, x_{i-L+1}\right]$, where $L$ is usually referred to as embedding dimension. This pattern is a point in the $L$-dimensional embedding space in which the dynamics of $x$ is reconstructed [20]. This sequence $x_{i}^{L}$ is constituted by the current sample $x_{i}$ and by the pattern $\boldsymbol{x}_{i-1}^{L-1}$ containing $L-1$ past samples of $x$ (i.e., $\left.\boldsymbol{x}_{i-1}^{L-1}=\left[x_{i-1}, \ldots, x_{i-L+1}\right]\right)$. Therefore, $\boldsymbol{x}_{i}^{L}$ can be indicated as $\boldsymbol{x}_{i}^{L}=\left[x_{i}, \boldsymbol{x}_{i-1}^{L-1}\right]$, thus stressing that the current value, $x_{i}$, may be conditioned by the previous $L-1$ values, $\boldsymbol{x}_{i-1}^{L-1}$, constituting the memory that might actually limit the unpredictability of future samples of $x$. After defining the set of all possible patterns as $\boldsymbol{x}^{L-1}=\left\{\boldsymbol{x}_{i-1}^{L-1}, i=L, \ldots, N\right\}$, the CE of $x$ given $x^{L-1}$, measuring the amount of information carried by $x_{i}$ that cannot be derived from $\boldsymbol{x}_{i-1}^{L-1}$, is computed as:

$$
\mathrm{CE}_{x \mid x^{L-1}}=-\sum p\left(x_{i}, \boldsymbol{x}_{i-1}^{L-1}\right) \cdot \log p\left(x_{i} \mid \boldsymbol{x}_{i-1}^{L-1}\right)
$$


where $p\left(x_{i}, \boldsymbol{x}_{i-1}^{L-1}\right)$ is the joint probability of pattern $\boldsymbol{x}_{i}^{L}$ and $p\left(x_{i} \mid \boldsymbol{x}_{i-1}^{L-1}\right)$ is the conditional probability of $x_{i}$ given $x_{i-1}^{L-1}$. The sE of $x$ set by $x^{L-1}$, quantifying the amount of information carried by $x$ that can be completely derived from $x^{L-1}$ is calculated as the mutual information between $x$ and $\boldsymbol{x}^{L-1}$ as:

$$
\mathrm{sE}_{x, x^{L-1}}=\sum p\left(x_{i}, \boldsymbol{x}_{i-1}^{L-1}\right) \cdot \log \frac{p\left(x_{i}, \boldsymbol{x}_{i-1}^{L-1}\right)}{p\left(x_{i}\right) \cdot p\left(\boldsymbol{x}_{i-1}^{L-1}\right)}
$$

where $p\left(x_{i}\right)$ is the probability of the sample $x_{i}$ and $p\left(x_{i-1}^{L-1}\right)$ is the probability of the pattern $\boldsymbol{x}_{i-1}^{L-1}$. The ShE of $x$, measuring the overall amount of information carried by $x$, is computed as:

$$
\operatorname{ShE}_{x}=-\sum p\left(x_{i}\right) \cdot \log p\left(x_{i}\right)
$$

It can be easily demonstrated [7-10] that:

$$
\mathrm{ShE}_{x}=\mathrm{CE}_{x \mid x^{L-1}}+\mathrm{sE}_{x, x^{L-1}}
$$

In this study CE was assessed as the corrected CE (CCE) [3]. The CCE exploits a coarse graining approach based on a uniform binning of $x$ over six quantization levels, thus producing a partition of the $L$-dimensional embedding space into hyper-cubes of side equal to the bin size. The CCE adopts a strategy to limit the loss of reliability of the CE estimate with L, the so-called curse of dimensionality [21], leading to a decrease of $\mathrm{CE}$ towards 0 regardless the type of the dynamics [3]. This strategy empirically raises the $\mathrm{CE}$ estimate towards the situation of maximum uncertainty quantified by $\mathrm{ShE}_{x}$ when $\log p\left(x_{i} \mid \boldsymbol{x}_{i-1}^{L-1}\right)=0$ is the result of the unique occurrence of $\boldsymbol{x}_{i-1}^{L-1}$ in the $(L-1)$-dimensional embedding space. This rise is regulated by the fraction of $x_{i-1}^{L-1}$ found only one time under the resolution adopted by the coarse graining procedure. According to the traditional setting utilized for the computation of MSCE and RMSCE, $L$ was fixed to 3 [2,11]. According to the RMSCE approach [12] at any iteration the Butterworth filter was exploited to eliminate fast temporal scales and uniform binning procedure was applied to the filterer version of $x$. At each iteration the bin size was computed as the difference between the maximum and the minimum of the filtered version divided by the number of bins. Equation (4) was utilized to estimate the sE after the computation of ShE and CE over the filtered version of $x$ at any time scale, thus obtaining the RMSsE. CE was approximated as CCE.

\section{Experimental Protocol and Data Analysis}

\subsection{Study Population and Signal Acquisition}

Forty-seven 12 leads 24-hour Holter surface electrocardiographic recordings (Mortara Instrument Inc., Milwaukee, WI, USA and Ela Medical, Sorin Group, Arvada, CO, USA) were acquired in this study from 47 individuals being a subgroup of a founder LQT1 population carrying the KCNQ1-A341V mutation [18,19]. The sampling rate was $180 \mathrm{~Hz}$ for Mortara recordings and $200 \mathrm{~Hz}$ for Ela Medical. Thirteen subjects (age from 27 to 56 years, median: 37 years, 6 males) were taken as a control group because they were non-MCs (NMCs), while the remaining 34 individuals were mutation carriers (MCs) divided into 11 AMCs (age from 24 to 62 years, median: 46 years, 4 males) and 23 SMCs 
(age from 16 to 57 years, median: 39 years, 9 males). Age and gender distributions were not different among groups. Subjects did not take any medications, included beta-blockers, i.e., the golden standard therapy for LQT1, at the time of recording. The protocol was approved by the ethical review board of the Universities of Stellenbosch and Pavia and adhered to the principles of the Declaration of Helsinki for medical research involving humans. All subjects or family members signed an informed written consent for clinical and genetic evaluations.

\subsection{Series Extraction and Data Analysis}

HP and QT beat-to-beat time series were extracted from each 24-hour Holter recording after choosing the lead with the best signal-to-noise ratio. HP was computed as the time distance between two consecutive R-wave peaks after fixing their location via parabolic interpolation [22]. QT was taken as the time distance between the R-wave interpolated apex and T-wave offset, delineated as the point where the absolute value of the first derivative on the T-wave downslope went below a threshold set as the $30 \%$ of the maximum absolute value, locally computed over each T-wave decline [22]. After the extraction of the HP and QT measures on a beat-to-beat basis, the two series were manually checked for values coming from ectopic beats or misdetections. These values were corrected through cubic spline interpolation. Corrections did not exceed $5 \%$ of the overall length of the sequence considered for analysis. HP and QT sequences, composed by 5000 consecutive measures, were selected at random during daytime, (DAY, from 2 to $6 \mathrm{PM}$ ) and nighttime (NIGHT, from 0 to 4 AM) in all the considered groups (i.e., NMC, AMC and SMC). Individual diaries were checked in order to make analyses more homogenous for type and intensity of physical activity during both DAY and NIGHT: more specifically, we avoided periods of sleeping or high intensity physical exercise during DAY and awake periods during NIGHT.

Traditional time domain parameters, such as the mean of HP, QT and corrected QT interval (QTc), evaluated according to the Bazett's formula [23], and the variance of HP and QT, were calculated, labeled as $\mu \mathrm{HP}, \mu \mathrm{QT}, \mu \mathrm{QTc}, \sigma^{2} \mathrm{HP}$ and $\sigma^{2} \mathrm{QT}$, and expressed as $\mathrm{ms}, \mathrm{ms}, \mathrm{ms}^{\cdot} \cdot \mathrm{s}^{-0.5}, \mathrm{~ms}^{2}$ and $\mathrm{ms}^{2}$ respectively. RMSCE and RMSsE were calculated over $x=$ HP and QT. According to [13] values of RMSCE and RMSsE in specific ranges of time scales were pooled together as follows: (i) at short time scale (i.e., $\tau=1)$, thus accounting for all frequency components present in the series (i.e., from 0 to $0.5 \mathrm{~Hz}$ ); (ii) at medium time scale (i.e., $2 \leq \tau \leq 4)$, thus progressively filtering fluctuations in the frequency band from $0.5 \mathrm{~Hz}$ to $0.125 \mathrm{~Hz}$ (with a HP mean of $1 \mathrm{~s}$ ) including the high frequency (HF, from 0.15 to $0.5 \mathrm{~Hz}$ ) components [24], and leaving almost untouched the oscillations in the low frequency (LF, from 0.04 to $0.15 \mathrm{~Hz}$ ) band [24]; (iii) at long time scale (i.e., $5 \leq \tau \leq 12$ ), thus progressively filtering oscillations from 0.125 to $0.042 \mathrm{~Hz}$ (with a HP mean of $1 \mathrm{~s}$ ) including not only the fluctuations in HF band but also those in LF band, and leaving intact only very LF oscillations (i.e., approximately those with frequencies slower than $0.042 \mathrm{~Hz}$ ).

\subsection{Statistical Analysis}

Paired t-test, or Wilcoxon signed rank test when appropriate, with correction for multiple comparisons was used to evaluate the significance of the DAY-NIGHT variations of RMSCE and RMSsE at a given time scale. After pooling temporal scales two way repeated measures analysis of 
variance (Holm-Sidak test for multiple comparisons, one factor repetition) was used to evaluate the significance of the change of RMSCE and RMSsE between experimental conditions (i.e., DAY versus NIGHT) within the same group and between groups (i.e., NMC, AMC and SMC) within the same experimental condition. The same test was utilized to check differences between time domain parameters. Linear correlation analysis was performed to measure the degree of association between RMSCE and RMSsE parameters computed at short (i.e., $\tau=1$ ), medium (i.e., $\tau=2-4$ ) and long (i.e., $\tau=5-12$ ) time scales in each group in each experimental condition. Pearson correlation coefficient, $r$, and type I error probability, $p$, were calculated. Statistical analysis was assessed with a commercial statistical program (Sigmaplot ver.11.0, Systat Software Inc, Chicago, IL, USA). Values were expressed as mean \pm standard deviation. A $p<0.05$ was always considered as significant.

\section{Results}

Table 1 shows results of time domain analyses of HP, QT and QTc. $\mu \mathrm{HP}, \sigma^{2} \mathrm{HP}, \mu_{\mathrm{QT}}, \sigma^{2} \mathrm{QT}$ and $\mu_{\mathrm{QT}}$ were computed in NMCs, AMCs and SMCs during DAY and NIGHT. $\mu_{\mathrm{HP}}$ was longer during NIGHT regardless of the population. Furthermore, AMCs were more bradycardic than NMCs during both DAY and NIGHT. On the contrary, $\sigma^{2}$ HP was similar in all groups and did not change during NIGHT. As a result of the pathology $\mu_{\mathrm{QT}}$ was longer in AMCs and SMCs during both DAY and NIGHT, and $\mu_{\mathrm{QT}}$ increased during NIGHT regardless of the group. During DAY $\sigma^{2} \mathrm{QT}$ in AMCs was larger than in NMCs and SMCs. In AMCs $\sigma^{2}$ QT decreased significantly during NIGHT. As expected $\mu_{\mathrm{QTC}}$ exhibited the same between-group modifications as $\mu_{\mathrm{QT}}$. In addition, we observed that $\mu_{\mathrm{QTc}}$ was smaller during NIGHT in both AMCs and SMCs.

Table 1. Time domain HP and QT indexes in NMCs, AMCs, SMCs during DAY and NIGHT.

\begin{tabular}{ccccccc}
\hline & \multicolumn{2}{c}{ NMC } & \multicolumn{2}{c}{ AMC } & \multicolumn{2}{c}{ SMC } \\
\cline { 2 - 6 } & DAY & NIGHT & DAY & NIGHT & DAY & NIGHT \\
\hline$\mu_{\mathrm{HP}}[\mathrm{ms}]$ & $694 \pm 112$ & $879 \pm 131^{*}$ & $830 \pm 161^{\S}$ & $1018 \pm 142^{*} \S$ & $757 \pm 94$ & $951 \pm 113^{*}$ \\
$\sigma_{\mathrm{HP}}^{2}\left[\mathrm{~ms}^{2}\right]$ & $1108 \pm 564$ & $2033 \pm 1659$ & $1651 \pm 1057$ & $1927 \pm 1595$ & $1520 \pm 1506$ & $2169 \pm 2151$ \\
$\mu_{\mathrm{QT}}[\mathrm{ms}]$ & $316 \pm 42$ & $356 \pm 29 *$ & $420 \pm 55^{\S}$ & $447 \pm 45^{*} \S$ & $407 \pm 43^{\S}$ & $445 \pm 29 * \S$ \\
$\sigma_{\mathrm{QT}}^{2}\left[\mathrm{~ms}^{2}\right]$ & $167 \pm 311$ & $82 \pm 79$ & $447 \pm 652^{\S}$ & $92 \pm 82^{*}$ & $132 \pm 84^{*}$ & $83 \pm 58$ \\
$\mu_{\mathrm{QTc}}\left[\mathrm{ms}^{-0.5} \mathrm{~s}^{-0.5}\right.$ & $380 \pm 30$ & $383 \pm 28$ & $462 \pm 34^{\S}$ & $445 \pm 31^{*}$, & $469 \pm 33^{\S}$ & $459 \pm 25^{*}$, \\
\hline
\end{tabular}

Note: Values are expressed as mean \pm standard deviation; * $p<0.05$ between DAY and NIGHT within the same group; $\$: p<0.05$ between AMCs or SMCs and NMCs within the same experimental condition (i.e., DAY or NIGHT); ${ }^{\#} p<0.05$ between AMCs and SMCs within the same experimental condition (i.e., DAY or NIGHT).

Figure 1 shows the RMSCE (Figure 1a,c,e) and RMSsE (Figure 1b,d,f), computed over HP in NMCs (Figure 1a,b), in AMCs (Figure 1c,d), and SMCs (Figure 1e,f) during DAY (black circles) and NIGHT (white circles). RMSCE of HP raised as a function of the time scale in NMCs, AMCs and SMCs (Figure 1a,c,e), while RMSsE of HP declined with time scale in all the groups (Figure 1b,d,f). RMSCE of HP tended to be higher during NIGHT at time scale $\tau=1$, and this finding was significant in AMCs (Figure 1c). At longer time scales the opposite situation was detected (i.e., RMSCE of HP was larger during DAY) and results were significant at time scales $\tau=7,9,12$ in NMCs (Figure 1a), $\tau=12$ in AMCs (Figure 1c) and $3 \leq \tau \leq 12$ in SMCs (Figure 1e). RMSsE of HP was higher during DAY at any 
scales and this finding was significant at short time scales and, more specifically, at $\tau=1,2$ in NMCs (Figure 1b), $\tau=1,2$ in AMCs (Figure 1d) and $1 \leq \tau \leq 3$ in SMCs (Figure 1f).
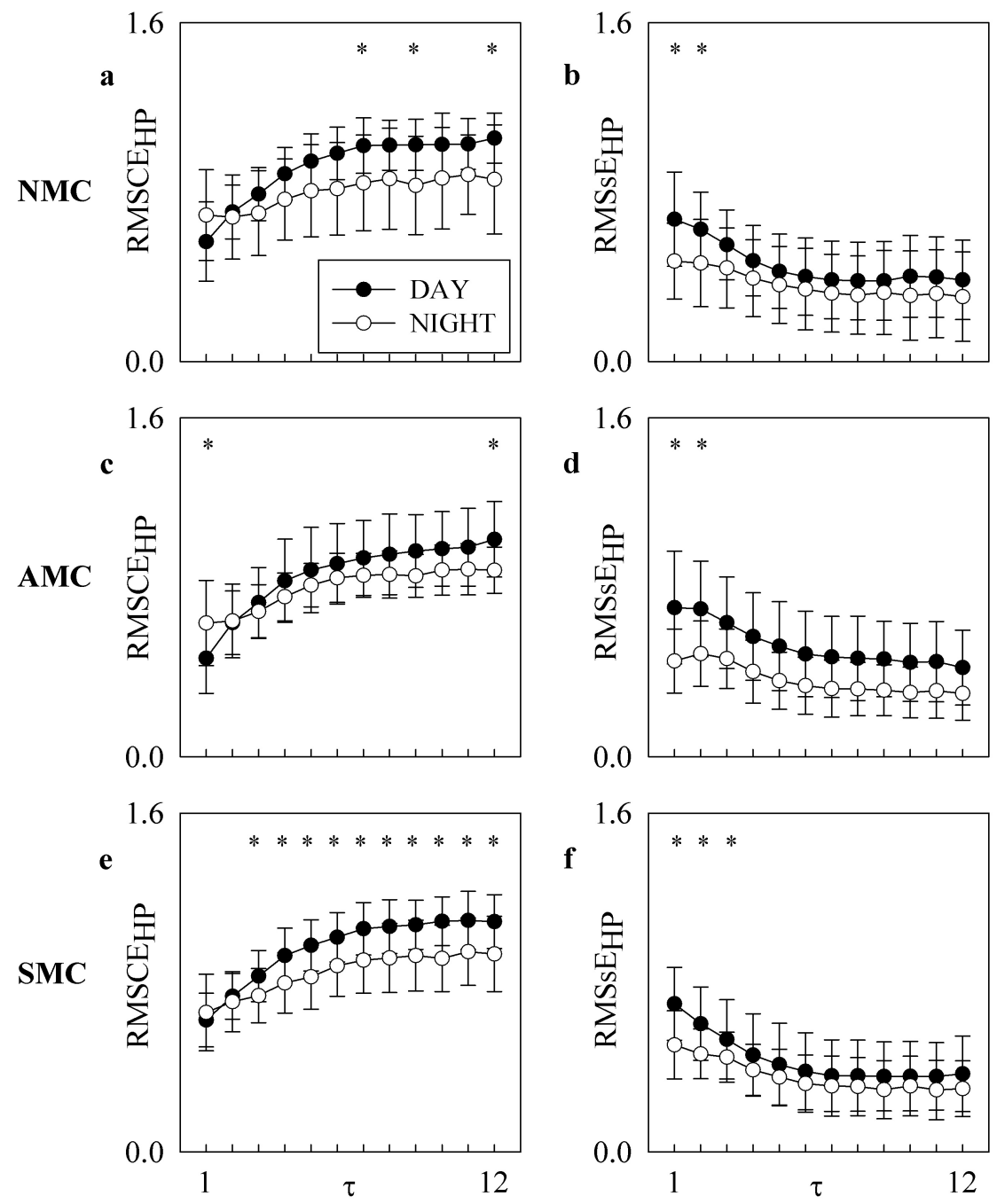

Figure 1. Multiple error line plots show RMSCE (a,c,e) and RMSsE (b,d,f) computed over HP in NMCs $(\mathbf{a}, \mathbf{b})$ in AMCs (c,d) and SMCs (e,f) during DAY (black circles) and NIGHT (white circles). Time scale $\tau$ ranges from 1 to 12 . Data are reported mean \pm standard deviation. The symbol * indicates $p<0.05$ between DAY and NIGHT at the corresponding time scale.

Figure 2 shows the RMSCE (Figure 2a,c,e) and RMSsE (Figure 2b,d,f) computed over QT in NMCs (Figure 2a,b), in AMCs (Figure 2c,d), and SMCs (Figure 2e,f) during DAY (black circles) and NIGHT (white circles). RMSCE of QT in NMCs and SMCs tended to reach a plateau after an initial decrease (Figure 2a,e), while the course of RMSCE of QT in AMCs was different (i.e., it increased steadily, Figure 2c). Also the RMSsE of QT raised with time scale and its course was similar across the groups (Figure 2b,d,f). The RMSCE of QT in AMCs and SMCs was significantly larger during DAY than NIGHT (Figure 2c,e) and this result was significant at the longest time scales (i.e., $3 \leq \tau \leq 12$ with $\tau \neq 5$ in AMCs and $2 \leq \tau \leq 12$ in SMCs). RMSCE of QT did not exhibit circadian variations in NMCs (Figure 2a). The RMSsE of QT increased steadily with time scale during both DAY and NIGHT and this result was consistently detected in all the considered groups (Figure 2b,d,f). 

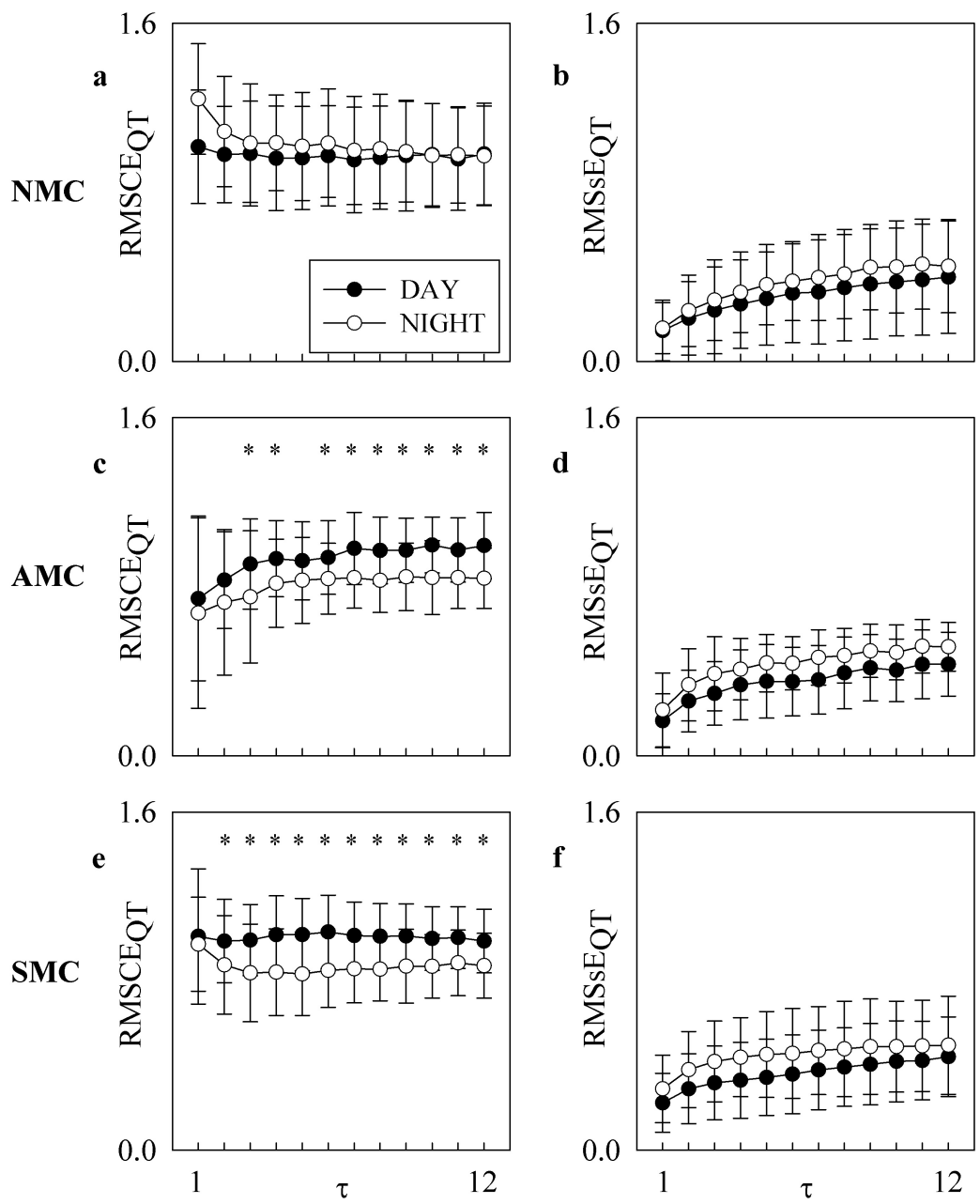

Figure 2. Multiple error bar line plots show RMSCE (a,c,e) and RMSsE (b,d,f) computed over QT in NMCs $(\mathbf{a}, \mathbf{b})$ in AMCs $(\mathbf{c}, \mathbf{d})$ and SMCs $(\mathbf{e}, \mathbf{f})$ during DAY (black circles) and NIGHT (white circles). Time scale $\tau$ ranges from 1 to 12 . Data are reported as mean \pm standard deviation. The symbol * indicates $p<0.05$ between DAY and NIGHT at the corresponding time scale.

Figure 3 shows RMSCE (Figure 3a,c,e) and RMSsE (Figure 3b,d,f) calculated over HP at short (Figure 3a,b), medium (Figure 3c,d) and long (Figure 3e,f) time scales as a function of the population (i.e., NMC, AMC and SMC) during DAY (back bars) and NIGHT (white bars). In HP series the RMSsE at short time scale was remarkable (Figure 3b), being of the same order of magnitude as RMSCE (Figure 3a), slightly reduced at medium time scale (Figure 3d) and significantly lower at long time scale (Figure 3f). At short time scale (i.e., $\tau=1$ ) RMSCE of HP was higher during NIGHT than DAY in all groups (Figure 3a) and this finding was significant only in AMCs. Conversely, at short time scale the RMSsE of HP featured the opposite situation with RMSsE of HP significantly higher during DAY than NIGHT in NMCs (Figure 3b). 

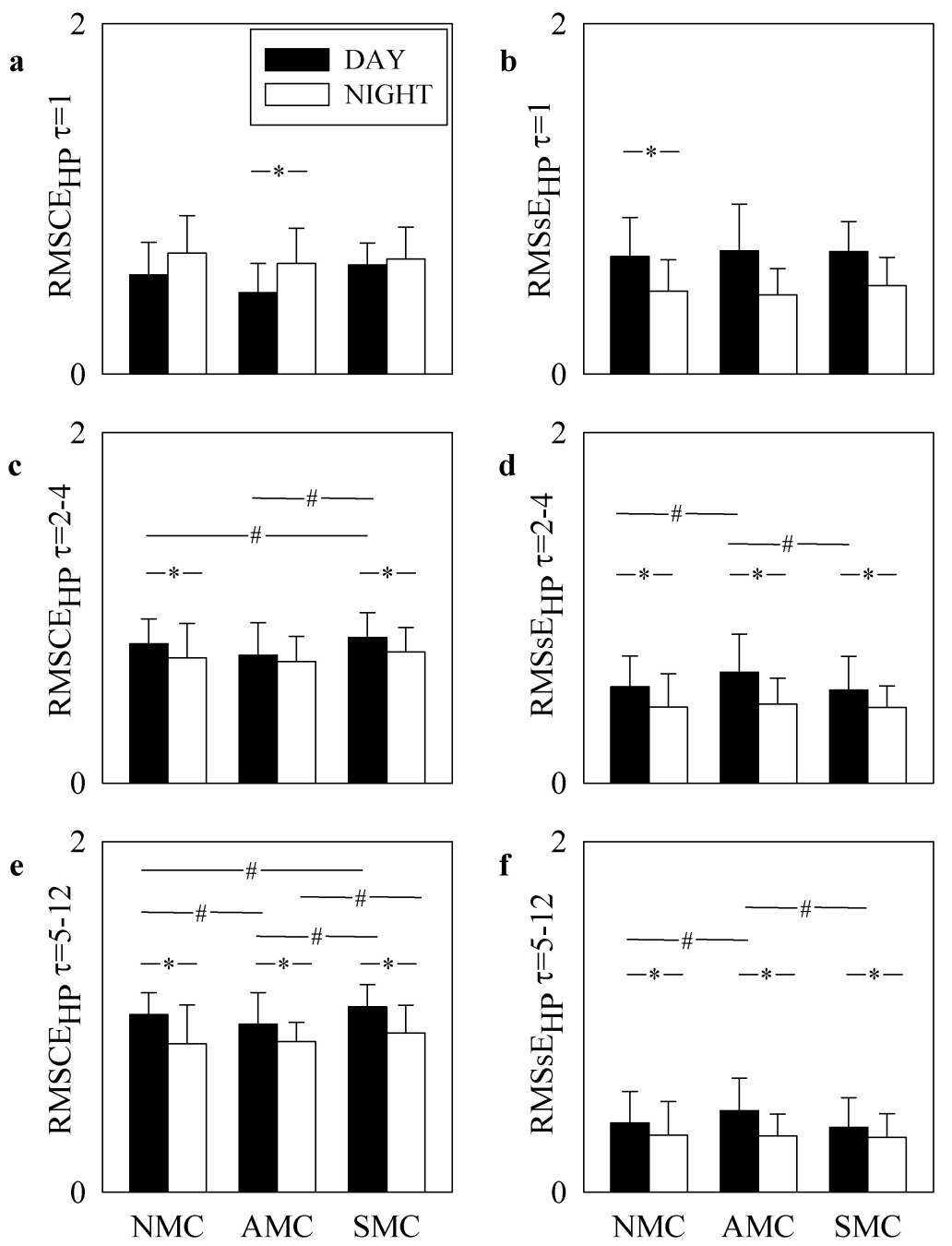

Figure 3. Grouped bar graphs show RMSCE (a,c,e) and RMSsE (b,d,f) calculated on HP at short $(\mathbf{a}, \mathbf{b})$, medium $(\mathbf{c}, \mathbf{d})$ and long $(\mathbf{e}, \mathbf{f})$ time scales as a function of the population (i.e., NMC, AMC and SMC) during DAY (back bars) and NIGHT (white bars). Data are reported mean + standard deviation. The symbol * indicates $p<0.05$ between DAY and NIGHT within the same group and the symbol \# denotes $p<0.05$ between groups within the same experimental condition.

At short time scale no difference was detected among groups given the same experimental condition (Figure 3a,b). At medium and long time scales both RMSCE and RMSsE of HP were larger during DAY than NIGHT and this result was significant in all the populations (Figure 3c-f) except in the case of the DAY-NIGHT comparison of RMSCE at medium temporal scales in AMCs (Figure 3c). During DAY the RMSCE of HP in SMCs was significantly larger than that of AMCs and NMCs at both medium and long time scales (Figure 3c,e) and at long time scale it was significantly smaller in AMCs than in NMCs (Figure 3e). During NIGHT the RMSCE of HP did not differentiate the groups except at long time scale where the RMSCE of HP in SMCs was found larger than that in AMCs (Figure 3e). Remarkably, RMSsE of HP differentiated groups at medium and long time scales with RMSsE significantly larger in AMCs than in both NMCs and SMCs during DAY (Figure 3d,f). 
Figure 4 shows RMSCE (Figure 4a,c,e) and RMSsE (Figure 4b,d,f) calculated over QT at short (Figure 4a,b), medium (Figure 4c,d) and long (Figure 4e,f) time scales as a function of the population (i.e., NMC, AMC and SMC) during DAY (back bars) and NIGHT (white bars).
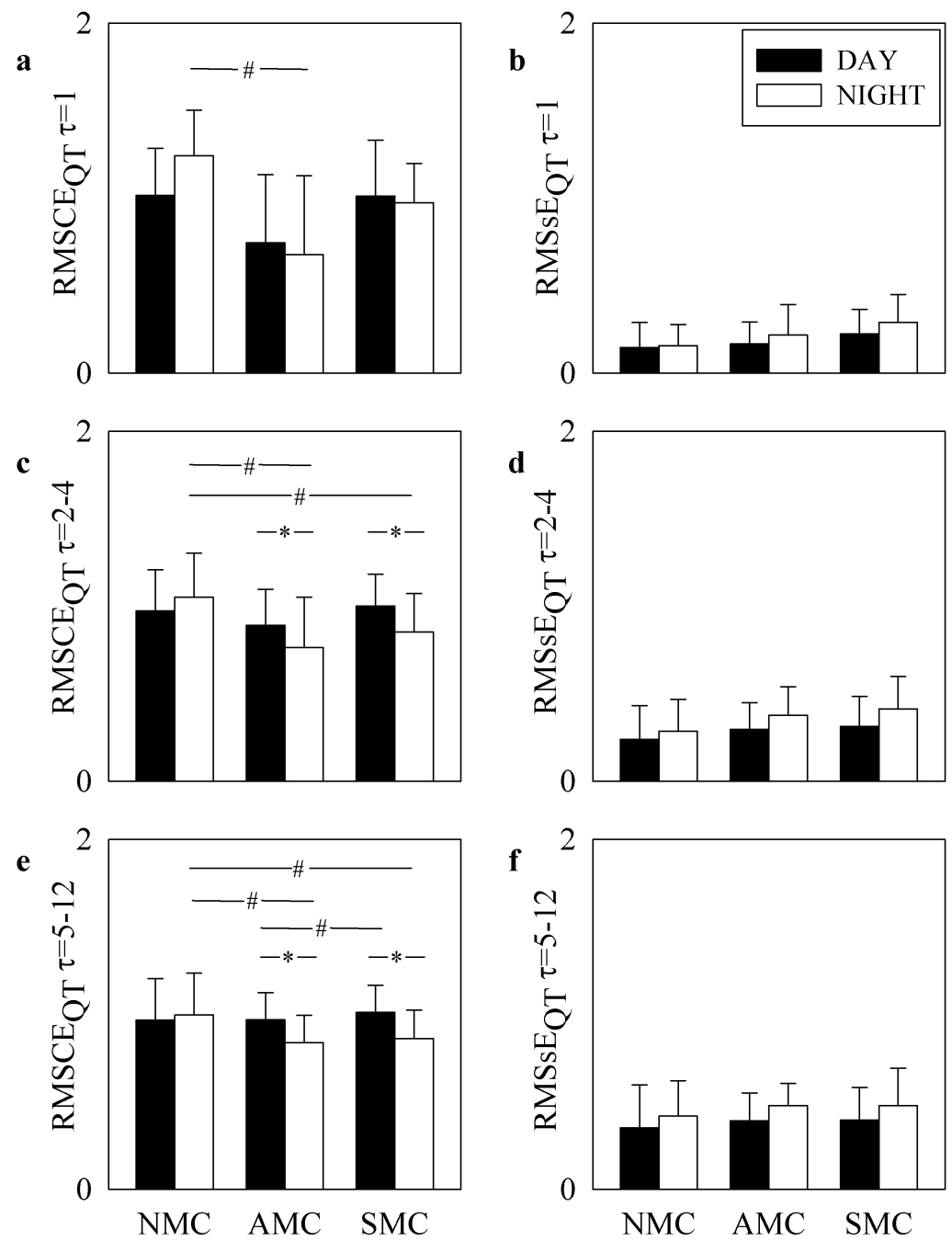

Figure 4. Grouped bar graphs show RMSCE (a,c,e) and RMSsE (b,d,f) calculated on QT at short $(\mathbf{a}, \mathbf{b})$, medium $(\mathbf{c}, \mathbf{d})$ and long $(\mathbf{e}, \mathbf{f})$ time scales as a function of the population (i.e., NMC, AMC and SMC) during DAY (back bars) and NIGHT (white bars). Data are reported mean + standard deviation. The symbol * indicates $p<0.05$ between DAY and NIGHT within the same group and the symbol \# denotes $p<0.05$ between groups within the same experimental condition.

In QT series the RMSsE was much smaller than RMSCE at short (Figure 4a,b), medium (Figure 4c,d) and long time scales (Figure 4e,f). RMSCE of QT exhibited a large inter-subject variability at short time scale (Figure 4a) that prevents a clear separation among groups and experimental conditions (only during NIGHT the RMSCE of QT in AMCs was significantly smaller than that in NMCs). Conversely, the RMSCE of QT was less variable at medium and long time scales, thus allowing the detection of significant circadian variations in AMCs and SMCs (Figure 4c,e) and the decrease in AMCs and SMCs compared to NMCs during NIGHT (Figure 4c,e). In addition, during DAY at long time scale RMSCE of QT increased in SMCs compared to AMCs (Figure 4e), while 
RMSsE of QT was less informative. Indeed, RMSsE could not distinguish groups and experimental conditions (Figure 4b,d,f).

Table 2 reports the Pearson correlation coefficient, $r$, and type I error probability, $p$, of the correlation analysis between RMSCE and RMSsE parameters at short (i.e., $\tau=1$ ), medium (i.e., $\tau=2-4$ ) and long (i.e., $\tau=5-12$ ) time scales in NMCs, AMCs and SMCs during DAY and NIGHT. Results of the correlation analysis over HP are first given and immediately followed by those over QT. It can be observed that RMSCE and RMSsE indexes were generally uncorrelated regardless of the time scale, experimental condition and group. The unique exceptions occurred in SMCs: indeed, the RMSCE and RMSsE parameters computed over HP at medium and long time scales during DAY and the RMSCE and RMSsE parameters calculated over QT at long time scale during DAY and NIGHT respectively were significantly correlated with $r<0$.

Table 2. Correlation analysis between RMSCE and RMSsE parameters evaluated over HP and QT at short, medium and long time scales in NMCs, AMCs and SMCs during DAY and NIGHT.

\begin{tabular}{ccccccc}
\hline & \multicolumn{2}{c}{$\begin{array}{c}\text { Short Time Scale } \\
\boldsymbol{\tau}\end{array}$} & \multicolumn{2}{c}{$\begin{array}{c}\text { Medium Time Scale } \\
\mathbf{2}\end{array}$} & \multicolumn{2}{c}{$\begin{array}{c}\text { Long Time Scale } \\
\mathbf{5} \leq \boldsymbol{\tau} \leq \mathbf{1 2}\end{array}$} \\
\cline { 2 - 7 } & $\boldsymbol{r}$ & $\boldsymbol{p}$ & $\boldsymbol{r}$ & $\boldsymbol{p}$ & $\boldsymbol{r}$ & $\boldsymbol{p}$ \\
\hline HP, NMC DAY & -0.277 & 0.359 & -0.12 & 0.696 & -0.389 & 0.189 \\
HP, AMC DAY & 0.0451 & 0.895 & 0.29 & 0.387 & 0.221 & 0.513 \\
HP, SMC DAY & -0.241 & 0.268 & $-0.623 *$ & $1.51 \cdot 10^{-3} *$ & $-0.686 *$ & $3.03 \cdot 10^{-4} *$ \\
HP, NMC NIGHT & 0.192 & 0.53 & 0.167 & 0.586 & 0.0502 & 0.871 \\
HP, AMC NIGHT & -0.0483 & 0.888 & -0.21 & 0.536 & -0.348 & 0.295 \\
HP, SMC NIGHT & -0.302 & 0.144 & -0.0358 & 0.871 & -0.273 & 0.208 \\
\hline QT, NMC DAY & -0.322 & 0.284 & -0.356 & 0.232 & -0.444 & 0.128 \\
QT, AMC DAY & 0.461 & 0.154 & -0.271 & 0.421 & -0.567 & 0.0687 \\
QT, SMC DAY & 0.0683 & 0.757 & -0.346 & 0.106 & $-0.553 *$ & $6.21 \cdot 10^{-3} *$ \\
QT, NMC NIGHT & -0.303 & 0.315 & -0.376 & 0.206 & -0.462 & 0.112 \\
QT, AMC NIGHT & -0.394 & 0.231 & -0.446 & 0.169 & -0.0189 & 0.956 \\
QT, SMC NIGHT & -0.225 & 0.301 & -0.213 & 0.328 & $-0.523 *$ & $1.04 \cdot 10^{-2} *$ \\
\hline
\end{tabular}

$r=$ Pearson correlation coefficient; $p=$ type I error probability; $*: p<0.05$.

\section{Discussion}

The present study proposes a multiscale approach of the two portions of the ShE, i.e., $\mathrm{CE}$ and $\mathrm{sE}$, with the major aim at characterizing the complexity of cardiac control and improving risk stratification in LQT1 via the analysis of HP and QT variabilities derived from 24-hour electrocardiographic recordings acquired routinely as a part of the monitoring of this pathological population.

The main findings can be summarized as follows: (i) RMSCE and RMSsE are worth to be monitored together as a function of the time scale because they provide non-redundant information about cardiac regulation; (ii) RMSsE differentiates experimental conditions (i.e., DAY and NIGHT) within the same population; (iii) RMSsE distinguishes healthy subjects from pathological individuals (i.e., NMCs from AMCs and SMCs) within the same experimental condition and separates groups according to the severity of the symptoms and arrhythmic risk (i.e., AMCs from SMCs). 


\subsection{RMSCE and RMSSE Carry Non-Redundant Information}

RMSCE, measuring the information carried by a series that cannot be derived from its own past, and RMSsE, quantifying the information carried by a series that is completely obtained from its own past, are non-redundant information-theoretic quantities. Indeed, their sum is the ShE of the series at any time scale (i.e., the overall amount of information carried by the series at a given temporal scale) [7-10]. Thus, RMSCE and RMSsE depend on changes of ShE and their relative balance across the experimental conditions, groups and time scales. The non-redundancy of these two quantities is confirmed by the general lack of correlation between RMSCE and RMSsE parameters (Table 2). In HP series the course of RMSCE and RMSsE seems to be complementary: the gradual increase of RMSCE with the time scale was associated to the progressive decrease of the RMSsE. In QT series this apparent relationship is absent: the variable course of RMSCE of QT as a function of the time scale (i.e., in NMCs and SMCs the RMSCE of QT showed an initial decrease at short time scales followed by a plateau, while in AMCs it exhibited a constant rise) was associated to patterns of RMSsE of QT more homogenous among groups and experimental conditions and characterized by a gradual rise with time scale.

In addition, in HP series both RMSCE and RMSsE exhibited a circadian pattern within the same group, thus indicating that both information-theoretic quantities can differentiate diverse states of the autonomic nervous system given the population, and can distinguish different groups assigned the experimental condition (i.e., DAY or NIGHT), thus suggesting that both quantities can typify pathological individuals given the experimental situation. Indeed, RMSCE of HP at long time scale and RMSsE of HP at medium and long time scales are larger during DAY than NIGHT regardless of the group and identify the peculiarity of AMCs compared to SMCs with RMSCE and RMSsE of HP respectively smaller and larger in AMCs than in NMCs and SMCs. This capability in separating DAY from NIGHT and AMCs from SMCs was preserved even when RMSCE of QT at long time scale was computed. Conversely, RMSsE of QT was useless at this regard. The different statistical power of the analysis carried out over HP and QT might be the result of the different proportion of RMSCE and RMSsE in the two series. Indeed, while in HP series RMSCE and RMSsE were of the same order of magnitude, the balance between RMSCE and RMSsE in QT series was largely in favor of RMSCE (i.e., RMSsE was quite small). We conclude that the information storage is much higher in the HP series than in the QT series. In the range of the time scales considered in this study a large variety of reflexes including baroreflex, cardiopulmonary circuits and direct central vagal and sympathetic influences actively regulate HP [25]. These reflexes and autonomic inputs are responsible for the rhythmical fluctuations of HP, usually quantified via spectral analysis [24], and for the large degree of predictability of the HP series, here quantified by RMSsE, especially under sympathetic activation $[8,14,15,26]$. Conversely, QT regulation is only partially mediated by HP via the QT-HP relation [27,28] and largely independent of sinus node regulation via neural inputs directly acting over the ventricles [29-37]. The QT regulation is highly complex involving differential modulations of the atria and ventricles, variable set points depending on the region of the heart and a more anatomically distributed network compared to the one of HP, mainly mediated by the autonomic innervation of sinus node, thus resulting in a smaller degree of predictability of the QT series and negligible QT information storage compared to HP, especially at short and medium time scales [13,14,38-40]. However, the higher 
complexity of QT at short time scale is compatible with the low signal-to-noise ratio characterizing its variability as well $[22,41]$. Remarkably, at long time scale the information storage of QT and HP were similar, thus supporting the observation that the high level of unpredictability of QT dynamics is due to fast components unexplained by past QT values and even by past HP samples.

\subsection{RMSCE and RMSSE Reflect DAY-NIGHT Variations}

It is well-known that the complexity of HP, as estimated by the RMSCE of HP, is under vagal control at short time scale $[12,13,15,42,43]$ and under sympathetic control at long time scale $[12,13,15]$. This study confirms these observations. Indeed, at short time scale RMSCE of HP tended to increase during NIGHT and at long time scale it was significantly larger during DAY. It was suggested that RMSCE of QT at long time scale is under sympathetic control [13,38]. This study supports only partially this conclusion. Indeed, the expected decrease of the RMSCE of QT during NIGHT at long time scale was detected only in AMCs and SMCs, while no circadian variations were observed in NMCs.

Originally, the present study monitored RMSsE in addition to RMSCE, thus allowing their first comparison. The RMSsE of HP was under sympathetic control being lower during NIGHT. This result was significant regardless of the group at medium and long time scales. This finding prompts for the exploitation of indexes quantifying information storage of HP as candidate markers for monitoring the state of the sympathetic control directed to the sinus node [5]. Since these markers do not depend on the amplitude of the HP fluctuations, mainly under vagal control in humans [44], they can be more powerful than spectral indexes of HP variability in monitoring the state of the sympathetic modulation. The information storage in the QT series, as quantified by the RMSsE of QT, was less powerful in detecting DAY-NIGHT variations as a likely result of the small amount of the QT information storage.

\subsection{RMSCE and RMSsE Distinguish LQT1 Individuals with Different Severity of Symptoms}

AMC and SMC correspond to two groups featuring completely different arrhythmic risk. Indeed, while AMCs have a lower risk to develop fatal events, SMCs are at higher risk and actually they have already experienced at least one risky event to be classified SMCs. Therefore, any parameters capable to distinguish these groups might be a good candidate to typify the arrhythmic risk in LQT1 population. We confirm that RMSCE of HP and QT at long time scale during DAY can distinguish AMCs from SMCs, being the complexity of HP and QT at long time scale in SMCs significantly higher than that of AMCs [13]. This finding suggested that having a reduced complexity of the cardiac control both at the level of sinus node and ventricles during DAY (i.e., when the arrhythmic risk is greatest in LQT1) is a protective factor [13]. Since AMCs are characterized by a more reactive sympathetic control [45] and a less reactive vagal regulation [45-47], this reduced complexity of the sympathetic control might be the result of the synchronization of neural inputs operating at slightly different temporal scales performed by the higher sympathetic driver [43,48]. However, this study added a new, relevant, piece of information. The RMSCE of HP at long time scale in AMCs during the period at the greatest arrhythmic risk (i.e., during DAY) was lower than that in NMCs, while SMCs did not differ from NMCs. This finding suggests that AMCs are also different from NMCs, thus allowing us to hypothesize that AMCs have a peculiar genetic make-up playing a significant role in limiting the severity of their disease and providing them a key advantage for survival with respect to SMCs. This conclusion 
supports previous observations about the differences of the sympathetic modulation among NMCs, AMCs and SMCs [45], reporting that the sympathetic control is higher in AMCs, while those of NMCs and SMCs are similar. It is noteworthy that traditional time domain indexes utilized in clinics $[18,19,46]$ cannot distinguish AMCs from SMCs (Table 1). Only a less frequently exploited time domain index such as the $\sigma^{2}$ QT can [45] (Table 1), thus suggesting that indexes derived from the multiscale complexity analysis are worth to be considered in addition to more traditional time domain parameters in clinics. We stress that the multiscale complexity analysis of HP is sufficient to separate AMCs and SMCs, thus rendering useless the extraction of the QT interval series and making the proposed analysis immediately applicable to HP series routinely extracted from 24-hour Holter recordings.

Remarkably, RMSsE of HP at long time scale during DAY accounted for the divergent arrhythmic risk of AMCs and SMCs as well. The information storage in HP at long time scale was higher in AMCs than in NMCs and SMCs, while NMCs and SMCs were indistinguishable at this regard. The higher level of HP predictability in AMCs might be again the effect of a more dominant sympathetic drive and a less reactive vagal control keeping high the information stored in HP, thus limiting the complexity of the HP control at long time scale. Furthermore, it is interesting to notice that a significant correlation between RMSCE and RMSsE indexes was found only in SMCs and more likely during DAY, thus suggesting a new peculiar feature (i.e., the significant association between unpredictability and information storage) that differentiates SMCs from NMCs and AMCs during the period of highest arrhythmic risk of LQT1 individuals (i.e., the DAY).

\section{Conclusions}

The study proposes an information-theoretic multiscale approach to assess the complexity of HP and QT series, as evaluated via RMSCE, and their information storage, as estimated via RMSsE. Such an approach was applied for the characterization of cardiac control and its relation with the arrhythmic risk in LQT1. Remarkably, the information stored into HP at long time scale was lower during NIGHT, as a likely result of the sympathetic withdrawal, and increased in AMCs compared to NMCs and SMCs, as a likely consequence of the higher sympathetic driver in AMCs. Since correlation between RMSCE and RMSsE parameters is generally absent, we recommend monitoring RMSsE in addition to RMSCE when the aim is the characterization of the cardiac control and risk profile in LQT1. The possible link of the information storage of HP with sympathetic control deserves further tests in healthy subjects during experimental maneuvers and physiological stimuli challenging cardiovascular control because, if confirmed, it might open new possibilities of monitoring autonomic function from HP variability by complementing indexes based on magnitude of HP variations (e.g., spectral markers) that are mainly under vagal control.

\section{Acknowledgments}

The authors would like to thank A.L. George Jr for the critical revision of the manuscript. 


\section{Author Contributions}

Vlasta Bari: analysis of the data, interpretation of the data, drafting the article, critical revision of the manuscript, final approval of the version to be published. Andrea Marchi and Beatrice De Maria: analysis of the data, final approval of the version to be published. Giulia Girardengo, Paul A. Brink, Lia Crotti and Peter J. Schwartz: acquisition of the data, critical revision of the manuscript, final approval of the version to be published. Alberto Porta: conception and design of the work, interpretation of the data, drafting the article, critical revision of the manuscript, final approval of the version to be published. All authors have read and approved the final manuscript.

\section{Conflicts of Interest}

The authors declare no conflict of interest.

\section{References}

1. Pincus, S.M. Approximate entropy as a measure of system complexity. Proc. Natl. Acad. Sci. USA 1991, 88, 2297-2311.

2. Richman, J.S.; Moorman, J.R. Physiological time-series analysis using approximate entropy and sample entropy. Am. J. Physiol. 2000, 278, H2039-H2049.

3. Porta, A.; Baselli, G.; Liberati, D.; Montano, N.; Cogliati, C.; Gnecchi-Ruscone, T.; Malliani, A.; Cerutti, S. Measuring regularity by means of a corrected conditional entropy in sympathetic outflow. Biol. Cybern. 1998, 78, 71-78.

4. Bandt, C.; Pompe, B. Permutation entropy: A natural complexity measure for time series. Phys. Rev. Lett. 2002, 88, 174102.

5. Porta, A.; Faes, L.; Nollo, G.; Bari, V.; Marchi, A.; de Maria, B.; Takahashi, A.C.M.; Catai, A.M. Conditional self-entropy and conditional joint transfer entropy in heart period variability during graded postural challenge. PLoS ONE 2015, 10, e0132851.

6. Lizier, J.T.; Pritam, S.; Prokopenko, M. Information dynamics in small-world networks. Artif. Life 2011, 17, 293-314.

7. Wibral, M.; Lizier, J.T.; Vogler, S.; Priesemann, V.; Galuske, R. Local active information storage as a tool to understand distributed neural information processing. Front. Neuroinformatics 2014, 8, doi:10.3389/fninf.2014.00001.

8. Porta, A.; Guzzetti, S.; Montano, N.; Pagani, M.; Somers, V.K.; Malliani, A.; Baselli, G.; Cerutti, S. Information domain analysis of cardiovascular variability signals: Evaluation of regularity, synchronisation and co-ordination. Med. Biol. Eng. Comput. 2000, 38, 180-188.

9. Lizier, J.T.; Prokopenko, M.; Zomaya, A.Y. Local measures of information storage in complex distributed computation. Inform. Sci. 2012, 208, 39-54.

10. Faes, L.; Porta, A.; Rossato, G.; Adami, A.; Tonon, D.; Corica, A.; Nollo, G. Investigating the mechanisms of cardiovascular and cerebrovascular regulation in orthostatic syncope through an information decomposition strategy. Auton. Neurosci. Basic Clin. 2013, 178, 76-82.

11. Costa, M.; Goldberger, A.L.; Peng, C.-K. Multiscale entropy analysis of complex physiologic time series. Phys. Rev. Lett. 2002, 89, 068102. 
12. Valencia, J.F.; Porta, A.; Vallverdù, M.; Clarià, F.; Baranowski, R.; Orlowska-Baranowska, E.; Caminal, P. Refined multiscale entropy: Application to 24-h Holter recordings of heart period variability in healthy and aortic stenosis subjects. IEEE Trans. Biomed. Eng. 2009, 56, 2202-2213.

13. Bari, V.; Valencia, J.F.; Vallverdù, M.; Girardengo, G.; Marchi, A.; Bassani, T.; Caminal, P.; Cerutti, S.; George, A.L.; Brink, P.A.; et al. Multiscale complexity analysis of the cardiac control identifies asymptomatic and symptomatic patients in long QT syndrome type 1. PLoS ONE 2014, 9, e93808.

14. Baumert, M.; Javorka, M.; Seeck, A.; Faber, R.; Sanders, P.; Voss, A. Multiscale entropy and detrended fluctuation analysis of QT interval and heart rate variability during normal pregnancy. Comput. Biol. Med. 2012, 42, 347-352.

15. Turianikova, Z.; Javorka, K.; Baumert, M.; Calkovska, A.; Javorka, M. The effect of orthostatic stress on multiscale entropy of heart rate and blood pressure. Physiol. Meas. 2011, 32, 1425-1437.

16. Humeau, A.; Mahé, G.; Chapeau-Blondeau, F.; Rousseau, D.; Abraham, P. Multiscale analysis of microvascular blood flow: A multiscale entropy study of laser Doppler flowmetry time series. IEEE Trans. Biomed. Eng. 2011, 58, 2970-2973.

17. Hu, J.; Gao, J.; Tung, W.-W.; Cao, Y. Multiscale analysis of heart rate variability: A comparison of different complexity measures. Ann. Biomed. Eng. 2010, 38, 854-864.

18. Brink, P.A.; Crotti, L.; Corfield, V.; Goosen, A.; Durrheim, G.; Hedley, P.; Heradien, M.; Geldenhuys, G.; Vanoli, E.; Bacchini, S.; et al. Phenotypic variability and unusual clinical severity of congenital long-QT syndrome in a founder population. Circulation 2005, 112, 2602-2610.

19. Brink, P.A.; Schwartz, P.J. Of founder populations, long QT syndrome, and destiny. Heart Rhythm 2009, 6, S25-S33.

20. Takens, F. Detecting strange attractors in fluid turbulence. In Dynamical Systems and Turbulence; Rand, D., Young, L.S., Eds.; Springer: Berlin, Germany, 1981; pp. 366-381.

21. Runge, J.; Heitzig, J.; Petoukhov, V.; Kurths, J. Escaping the curse of dimensionality in estimating multivariate transfer entropy. Phys. Rev. Lett. 2012, 108, 258701.

22. Porta, A.; Baselli, G.; Lombardi, F.; Cerutti, S.; Antolini, R.; Del Greco, M.; Ravelli, F.; Nollo, G. Performance assessment of standard algorithms for dynamic R-T interval measurement: Comparison between R-Tapex and R-Tend approach. Med. Biol. Eng. Comput. 1998, 36, 35-42.

23. Bazett, H.C. An analysis of the time-relations of electrocardiograms. Heart 1920, 7, 353-370.

24. Task Force of the European Society of Cardiology; The North American Society of Pacing and Electrophysiology. Heart rate variability - Standards of measurement, physiological interpretation and clinical use. Circulation 1996, 93, 1043-1065.

25. Koepchen, H.P. Physiology of rhythms and control systems: An integrative approach. In Rhythms in Physiological Systems; Haken, H., Koepchen, H.P., Eds.; Springer: Berlin, Germany, 1991; pp. 3-20.

26. Porta, A.; Baselli, G.; Guzzetti, S.; Pagani, M.; Malliani, A.; Cerutti, S. Prediction of short cardiovascular variability signals based on conditional distribution. IEEE Trans. Biomed. Eng. 2000, 47, 1555-1564.

27. Molnar, J.; Weiss, J.S.; Rosenthal, J.E. The missing second: What is the correct unit for the Bazett corrected QT interval? Am. J. Cardiol. 1995, 75, 537-538. 
28. Pueyo, E.; Smetana, P.; Caminal, P.; de Luna, A.B.; Malik, M.; Laguna, P. Characterization of QT interval adaptation to RR interval changes and its use as a risk-stratifier of arrhythmic mortality in Amiodarone-treated survivors of acute myocardial infarction. IEEE Trans. Biomed. Eng. 2004, $51,1511-1520$.

29. Ahnve, S.; Vallin, H. Influence of heart rate and inhibition of autonomic tone on the QT interval. Circulation 1982, 65, 435-439.

30. Magnano, A.R.; Holleran, S.; Ramakrishnan, R.; Reiffel, J.A.; Bloomfiled D.M. Autonomic nervous system influences on QT interval in normal subjects. J. Am. Coll. Cardiol. 2002, 39, 1820-1826.

31. Shimizu, W.; Tsuchioka, Y.; Karawana, S.; Nagata, K.; Mukai, J.; Yamagata, T.; Matsuura, H.; Kajiyama, G.; Matsuura, Y. Differential effect of pharmacological autonomic blockade on some electrophysiological properties of the human ventricle and atrium. Br. Heart J. 1994, 71, 34-37.

32. Porta, A.; Baselli, G.; Caiani, E.; Malliani, A.; Lombardi, F.; Cerutti, S. Quantifying electrocardiogram RT-RR variability interactions. Med. Biol. Eng. Comput. 1998, 36, 27-34.

33. Negoescu, R.; Dinca-Panaitescu, S.; Filcescu, V.; Ionescu, D.; Wolf, S. Mental stress enhances the sympathetic fraction of QT variability in an RR-independent way. Integr. Phys. Beh. Sci. 1997, 32, 220-227.

34. Rickards, A.F.; Norman, J. Relation between QT interval and heart rate. New design of physiologically adaptive cardiac pacemaker. Br. Heart J. 1981, 45, 56-61.

35. Milne, J.R.; Ward, D.E.; Spurrell, R.A.; Camm, A.J. The ventricular paced QT interval—The effects of rate and exercise. Pacing Clin. Electrophysiol. 1982, 5, 352-358.

36. Browne, K.F.; Prystowsky, E.; Heger, J.J.; Zipes, D.P. Modulation of Q-T interval by the autonomic nervous system. Pacing Clin. Electrophysiol. 1983, 6, 1050-1055.

37. Porta, A.; Tobaldini, E.; Gnecchi-Ruscone, T.; Montano, N. RT variability unrelated to heart period and respiration progressively increases during graded head-up tilt. Am. J. Physiol. 2010, 298, H1406-H1414.

38. Bari, V.; Marchi, A.; de Maria, B.; Girardengo, G.; George, A.L.; Brink, P.A.; Cerutti, S.; Crotti, L.; Schwartz, P.J.; Porta, A. Low-pass filtering approach via empirical mode decomposition improves short-scale entropy-based complexity estimation of QT interval variability in long QT syndrome type 1 patients. Entropy 2014, 16, 4839-4854.

39. Lewis, M.J.; Short, A.L. Sample entropy of electrocardiographic RR and QT time-series data during rest and exercise. Physiol. Meas. 2007, 28, 731-744.

40. Bar, K.-J.; Koschke, M.; Berger, S.; Schulz, S.; Tancer, M.; Voss, A.; Yeragani, V.K. Influence of Olanzapine on QT variability and complexity measures of heart rate in patients with schizophrenia. J. Clin. Psychopharm. 2008, 28, 694-698.

41. Baumert, M.; Starc, V.; Porta, A. Conventional QT variability measurement vs. template matching techniques: Comparison of performance using simulated and real ECG. PLoS ONE 2012, 7, e41920.

42. Porta, A.; Faes, L.; Masé, M.; D'Addio, G.; Pinna, G.D.; Maestri, R.; Montano, N.; Furlan, R.; Guzzetti, S.; Nollo, G.; et al. An integrated approach based on uniform quantization for the evaluation of complexity of short-term heart period variability: Application to $24 \mathrm{~h}$ Holter recordings in healthy and heart failure humans. Chaos 2007, 17, 015117. 
43. Porta, A.; Gnecchi-Ruscone, T.; Tobaldini, E.; Guzzetti, S.; Furlan, R.; Montano, N. Progressive decrease of heart period variability entropy-based complexity during graded head-up tilt. J. Appl. Physiol. 2007, 103, 1143-1149.

44. Pomeranz, B.; Macaulay, R.J.B.; Caudill, M.A.; Kutz, I.; Adam, D.; Gordon, D.; Kilborn, K.M.; Barger, A.C.; Shannon, D.C.; Cohen, R.J.; Benson, H. Assessment of autonomic function in humans by heart-rate spectral-analysis. Am. J. Physiol. 1985, 248, H151-H153.

45. Porta, A.; Girardengo, G.; Bari, V.; George, A.L.; Brink, P.A.; Goosen, A.; Crotti, L.; Schwartz P.J. Autonomic control of heart rate and QT variability influences arrhythmic risk in long QT syndrome type 1. J. Am. Coll. Cardiol. 2015, 65, 367-374.

46. Schwartz, P.J.; Vanoli, E.; Crotti, L.; Spazzolini, C.; Ferrandi, C.; Goosen, A.; Hedley, P.; Heradien, M.; Bacchini, S.; Turco, A.; et al. Neural control of heart rate is an arrhythmia risk modifier in long QT syndrome. J. Am. Coll. Cardiol. 2008, 51, 920-929.

47. Crotti, L.; Spazzolini, C.; Porretta, A.P.; Dagradi, F.; Taravelli, E.; Petracci, B.; Vicentini, A.; Pedrazzini, F.; La Rovere, M.T.; Vanoli, E.; et al. Vagal reflexes following an exercise stress test: A simple clinical tool for gene-specific risk stratification in the long QT syndrome. J. Am. Coll. Cardiol. 2012, 60, 2515-2524.

48. Baselli, G.; Porta, A.; Pagani, M. Coupling arterial windkessel with peripheral vasomotion: Modeling the effects on low-frequency oscillations. IEEE Trans. Biomed. Eng. 2006, 53, 53-64.

(C) 2015 by the authors; licensee MDPI, Basel, Switzerland. This article is an open access article distributed under the terms and conditions of the Creative Commons Attribution license (http://creativecommons.org/licenses/by/4.0/). 